\title{
STANDORTSFAKTOREN UND BODENBILDUNG IN EINEM SUBALPINEN LAWINENANRISSGEBIET (STILLBERG BEI DAVOS)
}

\author{
P. BLASER
}

\section{Abstract:}

Soil formation in a deforested avalanche area at timber line near Davos was investigated by means of analysis of site factors. It is shown that there exist causal relations between ground relief, microclimate, vegetation and soil formation. Soil formation is discussed with respect to four characteristic sites of an avalanche couloir.

Das Versuchsgelände, in welchem die ökologisch bedeutsamen Standortsfaktoren für eine Aufforstung an der oberen Waldgrenze untersucht werden, liegt am linken, NE-exponierten Hang des Dischmatales in einer Höhe zwischen 2000 und $2230 \mathrm{~m}$ ü.M. Klimatisch liegt das Tal im Gebiet der Gebirgskontinentalen Hochalpen. Mit einer mittleren Jahrestemperatur von $1.6^{\circ} \mathrm{C}$ (Januarmittel: $-6.1^{\circ} \mathrm{C}$; Julimittel: $8.9^{\circ} \mathrm{C}$ ) und mittleren Jahresniederschlägen von $1240 \mathrm{~mm}$ (Periode 1901-1960) weist das Gebiet borealen Klimacharakter auf und liegt, auf die Bodenbildung bezogen, im Verbreitungsgebiet der Podsole.

Geologisch gehört das Dischmatal zum kristallinen Deckenmassiv der Silvretta.

Der Aufbau ist in diesem Talabschnitt recht einheitlich und besteht vorwiegend aus kristallinen Schiefer- und Gneisformationen.

Als Klimaxvegetation wird der Arven-Lärchenwald angesehen. Die potentielle Waldgrenze liegt in diesem Gebiet bei ca. $2250 \mathrm{~m}$, die heutige Waldgrenze dagegen bei ca. 2000 m ü.M., so dass angenommen werden kann, dass die Versuchsfläche ursprünglich bewaldet gewesen ist. Heute ist das Gebiet grösstenteils mit verschiedenen Zwergstrauchgesellschaften aus der oberen Zone des subalpinen Gürtels bewachsen.

Das Versuchsgelände liegt mitten in einem steilen Lawinenanriss mit 9 Lawinenzügen. Die mittlere Hangneigung beträgt $80 \%$. Das Relief ist sehr stark gegliedert. Die Fläche weist eine Anzahl markanter Geländerippen und Hangrunsen auf, in welche die nieder gehenden Lawinen kanalisiert werden. Diese ausgeprägte Geländegliederung bewirkt eine starke Differenzierung des Mikroklimas, durch welche die Verteilung der verschiedenen Pflanzengesellschaften in der Fläche teilweise erklärt werden kann. Da die ausgleichende Wirkung einer Baumschicht fehlt, kommt dem Relief als unabhängigem Standortsfaktor eine besondere Bedeutung zu. Bedingt durch die Talorientierung sind am NE-exponierten Stillberg in den 5-10 m tiefen postglazialen Geländerinnen N und E exponierte Nebenhangrichtungen typisch. Diese N-und E-Einhänge weisen während der Vegetationsperiode gegenüber anderen Expositionen sehr grosse Bestrahlungsunterschiede auf.

Wie die Untersuchungen von TURNER (1966) und NÄGELI (1971) zeigen, sind beinahe alle Klimafaktoren wie globale Hangstrahlung, Temperatur der bodennahen Luftschicht, Bodentemperatur, Wind usw. direkt vom Relief abhängig. Ebenso 
werden direkt, oder indirekt über das Mikroklima, die Dauer der Schneebedeckung, die Ausaperung, die Lawinentätigkeit sowie die Verteilung der Pflanzengesellschaften durch das Relief entscheidend beeinflusst (KUOCH \& AMIET, 1970). Auch die Verbreitung der verschiedenen Bodenformen macht hiervon keine Ausnahme. Diese ausgeprägte, reliefbedingte standörtlichökologische Differenzierung ist an einem Querprofil durch eine Hangkrete schematisch dargestellt, Abb. 1.

\section{Allgemeines über die Bodenbildung in der Versuchsfläche}

Wie eingangs erwähnt, liegt die Versuchsfläche klimatisch und geologisch in einem Gebiet, in dem die Podsolbildung vorherrscht. Podsole sind Böden, die bei intensiver Silikatverwitterung einer starken Abwärtsverlagerung von Eisen, Aluminium und

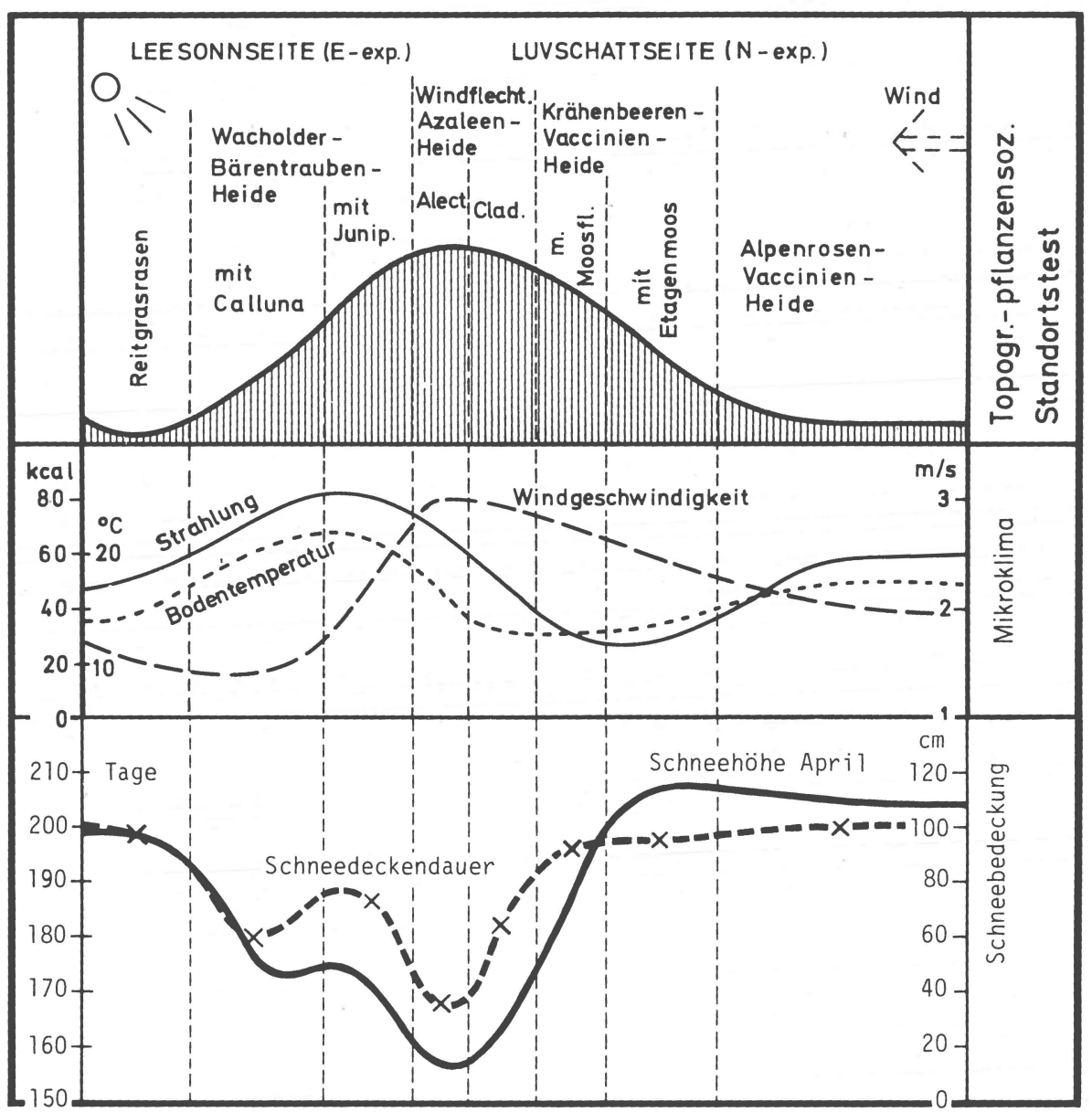

$A b b$. 1. Oekographische Uebersicht über Relief, Vegetation und klimatische Standortsfaktoren im Versuchsgelände Stillberg (nach TURNER et al., 1975). 
von organischen Humusstoffen unterliegen. Sehr oft besitzen solche Böden eine Rohhumusauflage.

Die Verlagerung von pedogenen Eisenverbindungen und von Humussubstanzen führt vielfach zu einer morphologisch sichtbaren Bleichung im Oberboden und zu einer Anreicherung von organischen und organometallischen Verbindungen im Unterboden, die mit einer deutlichen Schwarz- und Rostrotverfärbung einhergeht. Für die systematische Gliederung der podsolierten Böden wird in fast allen Fällen die Intensität der Bleichung in den Auswaschungshorizonten herangezogen, welche das Ausmass der Verlagerung insbesonders von Eisen und Humusverbindungen widerspiegelt. Zur genaueren Erfassung der Verlagerungsintensität werden auch chemische Kennwerte benutzt. Zur Charakterisierung der Podsolierung haben sich die Verlagerungsindices für Eisen, Aluminium und von Fulvosäuren bewährt (DUCHAUFOUR \& SOUCHIER 1963) welche ein relatives Mass für die Auswaschung dieser Verbindungen darstellen.

Die Verlagerungsprozesse im Boden werden durch verschiedene Standortsfaktoren entscheidend beeinflusst. So ist die Verlagerung des für die morphologische Differenzierung und für die systematische Zuordnung wichtigen Eisens an ganz bestimmte Voraussetzungen gebunden. Da unter aeroben Verhältnissen das Eisen in der dreiwertigen Form vorliegt und dadurch im $\mathrm{pH}$-Bereich $>3$ praktisch unlöslich ist, kann eine wirksame Verlagerung nur dann stattfinden, wenn sich relativ stabile Verbindungen mit wasserlöslichen organischen Stoffen bilden können, die über einen weiteren $\mathrm{pH}$-Bereich löslich sind. Organische Stoffe, die zur Komplexbildung mit Eisen befähigt sind, werden in erster Linie beim Streuabbau aus den Vegetationsrückständen freigesetzt; daneben werden aber auch aus den Humushorizonten ähnliche Verbindungen nachgeliefert, die aber ein wesentlich geringeres Eisenbindungsvermögen besitzen (BLOOMFIELD 1964).

Da das Eisenbindungsvermögen sowie die Stabilität solcher organischen Eisenverbindungen stark von der Art der pflanzlichen Streu abhängig ist, kommt der Vegetation durch die Art und Menge der von ihr gelieferten Streu bezüglich der Podsolierung eine grosse Bedeutung zu.

Solche wasserlösliche, organische Verbindungen mit unterschiedlichem Eisenbindungsvermögen können aber nur dann wirksam verlagert werden, wenn genügend Sickerwasser zur Verfügung steht, wenn sie nicht durch grössere Mengen tauschbarer Basen neutralisiert werden und wenn der mikrobielle Abbau während längerer Zeit unterbleibt.

Daraus wird ersichtlich, dass neben der Vegetation besonders das Klima sowie der Chemismus des Muttergesteins die Podsolierung stark beeinflussen. Alle Faktoren, welche die mikrobielle Aktivität hemmen, begünstigen indirekt die Podsolierung. Podsole sind darum die Klimaxböden der kühl-feuchten Klimate auf basenarmen Gesteinen.

\section{Die Bodenbildung an vier Hauptstandorten in der Versuchsfläche}

An vier charakteristischen Standorten in einem Lawinenzug wurden die Böden untersucht und eine Interpretation der Bodenbildung anhand der Standortsanalyse vorgenommen. In Tab. 1 und Abb. 1 sind die wichtigsten Resultate zusammengefasst. 
Tabelle 1. Standortsfaktoren und Bodenbildung

\begin{tabular}{lllll} 
Standort & Krete & Schattenhang & Runse & Sonnenhang \\
\hline Vegetation & $\begin{array}{l}\text { Cetrario- } \\
\text { loiseleurietum }\end{array}$ & $\begin{array}{l}\text { Empetro-Vaccinietum } \\
\text { hylocomietosum }\end{array}$ & $\begin{array}{l}\text { Calamagrostietum } \\
\text { villosae }\end{array}$ & $\begin{array}{l}\text { Junipero- } \\
\text { Arctostaphyletum }\end{array}$ \\
$\begin{array}{l}\text { 1. Rel. Eisenbindungsvermögen } \\
\text { der wasserlösl. Streusubstanz }\end{array}$ & 1.80 & 1.51 & 1.64 & 1.00 \\
$\begin{array}{l}\text { 1. Rel. Verlagerungstiefe } \\
\begin{array}{l}\text { 2. Globale Hangbestrahlung } \\
\text { kcal/cm }\end{array}\end{array}$ & 1.5 & 1.0 & 2.5 & 1.0 \\
$\begin{array}{l}\text { 3. Summe der Monatsmittel der } \\
\text { täglichen }\end{array}$ & $50-60$ & $10-20$ & $50-60$ & $70-90$ \\
$\begin{array}{l}\text { Temperaturmaxima } \\
\text { Bodemperaturminima }\end{array}$ & -15.2 & & & 116.4 \\
$\begin{array}{l}\text { Anzahl Monate/Jahr } \\
\text { mit Mittelwerten }>0^{\circ} \mathrm{C}\end{array}$ & 59.8 & 62.3 & 70.3 & 12.2 \\
$\begin{array}{l}\text { 4. Windgeschwindigk. verteilung, } \\
\text { \% des Flächenmittels }\end{array}$ & 150 & 4.4 & 16.1 & 9 \\
Boden & Flachgründige & Eisenhumus- & 9 & 120 \\
\hline
\end{tabular}

1. BLASER 1975; 2. Summe für die Vegetationsperiode 15. Mai-30. Sept. (TURNER 1966);

3. TURNER et al. 1975, (Mittelwerte der Jahre 1968-1970); 4. NÄGELI 1971 (Geschwindigkeit des Tagwindes in 1 m Höhe über der Bodenoberfläche). 
Die windgefegten Kuppen und Kreten sind während des Winters oft schneefrei und dadurch starkem Frost ausgesetzt. Unter diesen extremen Bedingungen kommt die Windflechten-Azaleenheide zur Vorherrschaft, deren Streu vergleichsweise ein grosses Podsolierungspotential besitzt. Bedingt durch die Lage im Relief, die starke Bewindung und die langen Perioden, während denen der Boden gefroren bleibt, handelt es sich um edaphisch trockene Standorte. Die Podsolierung verläuft an diesen Standorten sehr intensiv, die podsolierende Wirkung der Vegetation wird aber durch die Trockenheit eingeschränkt. Die charakteristischen Böden dieser Standorte sind hochentwickelte aber flachgründige Eisenhumus-Podsole mit geschlossenen Rohhumusauflagen.

An den N bis NNW exponierten Schatteneinhängen, welche die geringste Einstrahlung erhalten, kommt eine sehr moosreiche Variante der Krähenbeeren-Vaccinienheide zur Vorherrschaft. Unter den geschlossenen Moosteppichen bildeten sich extreme Rohhumusauflagen unter denen der Boden nie austrocknet. Bedingt durch die niedrigen Temperaturen vor allem in den Humushorizonten sowie durch das tiefe $\mathrm{pH}$ dürfte die biologische Aktivität stark gehemmt sein, wodurch das nicht sehr grosse Podsolierungspotential (Tab. 1) der wasserlöslichen Streusubstanz weitgehend erhalten bleibt. Die Böden dieser Standorte sind deutlich bis stark podsoliert und durch extreme Rohumusauflagen gekennzeichnet.

Die ökologische Besonderheit der Runsenstandorte besteht darin, dass der Boden während des ganzen Jahres praktisch nicht gefriert und dass die Temperaturminima auch unmittelbar an der Bodenoberfläche nur während ca. 3 Monaten des Jahres um einige Zehntelgrade unter dem Gefrierpunkt liegen. Diese Anomalie ist nicht durch die Einstrahlung bedingt, die etwa gleich gross ist wie auf den Kretenstandorten, sondern sie ist mit grosser Wahrscheinlichkeit auf den horizontalen Bodenwärmefluss zurückzuführen. Unter den speziellen standörtlichen Verhältnissen, besonders durch die direkten und indirekten Einflüsse der Lawinentätigkeit sind die Runsensohlen vom Reitgrasrasen besiedelt. Beim Streuabbau, der sehr intensiv erfolgt (beinahe totaler Abbau innert Jahresfrist), werden wasserlösliche Substanzen freigesetzt, die vergleichsweise ein sehr grosses Podsolierungsvermögen besitzen. Dieses Podsolierungspotential der Vegetation wird durch die speziellen Standortsverhältnisse noch wesentlich verstärkt. Da diese Böden im Gegensatz zu allen anderen untersuchten Standorten nicht gefrieren, kann das Wasser in diesen Sammelkanälen, besonders mit dem Eintreten der Schneeschmelze ungehindert durch den ohnehin schon sehr durchlässigen Boden versickern. Während der langen Nass-Kalt-Phasen, die sich bis weit in den Vorsommer hinziehen können, bleibt die Aktivität der Bodenorganismen infolge der ungünstigen Temperatur gehemmt, so dass die wasserlöslichen Verbindungen dem Abbau entgehen und mit dem Sickerwasser in die Tiefe verlagert werden.

Die Runsenstandorte unter dem Reitgrasrasen sind durch ausserordentlich tief ausgewaschene Böden gekennzeichnet. Die Bleichung der Eluvialhorizonte dürfte durch die gleichen, den Podsolen eigenen Prozesse entstanden sein, obwohl gerade diese Böden keine Rohhumusformen sondern einen oligotrophen Mull aufweisen.

Die E bis ESE exponierten Sonneneinhänge erfahren die intensivste Bestrahlung aller untersuchten Standorte der Versuchsfläche. Bedingt durch die thermische Begünstigung werden diese Einhänge von der wärmeliebenden, oft durch lockeren Wuchs gekennzeichneten Wacholder-Bärentraubenheide besiedelt. Die podsolierenden Eigenschaften der wasserlöslichen Streusubstanzen dieser Vegetation sind im 
Vergleich mit den anderen Gesellschaften am geringsten. Dieses kleine Podsolierungsvermögen der Vegetation wird durch die speziellen Standortsverhältnisse zusätzlich noch reduziert. So sind die Bedingungen für eine erhöhte bodenbiologische Aktivität besser als an den anderen Standorten. Der Abbau der aktiven organischen Verbindungen dürfte daher trotz zeitweiliger Ueberhitzung und oberflächlicher Austrocknung rascher vor sich gehen. Die Standorte sind zudem edaphisch eher trocken, so dass eine wirksame Verlagerung weitgehend verhindert wird. Die Böden der Sonneneinhänge sind allgemein wenig podsoliert. Oft fehlt ein morphologisch sichtbarer Bleichhorizont und die analytisch nachweisbare Verlagerung von Eisen und Aluminium bleibt unter der maskierenden schwarzen Farbe der mächtigen alpinen Moderformen weitgehend verborgen.

\section{LITERATUR}

BLASER, P. (1974): Mechanismen der Eisenaufnahme und -verlagerung durch wasserlösliche Streusubstanzen in podsoligen Böden. Mitt. deutsche Bodenkundl. Ges. 20: 447-457.

BLOOMFIELD, C. (1964): Organic matter and soil dynamics. In: Experimental pedology. Proceedings of the University of Nottingham, Easter school in agricultural science. 11: 257-266.

DUCHAUFOUR, Ph. \& SOUCHIER, B. (1963): Note sur un problème de classification Podsolisation chimique et différenciation du profil. Pédologie, XV, 2: 143-158.

KUOCH, R. \& AMIET, R. (1970): Die Verjüngung im Bereich der oberen Waldgrenze der Alpen. Mitt.schweiz. Anst.forstl. Vers'wes. 46: 4:159-328.

NÄGELI, W. (1971): Der Wind als Standortsfaktor bei Aufforstungen in der subalpinen Stufe (Stillberg im Dischmatal, Kanton Graubünden). Mitt.scbweiz.Anst.forstl.Vers'wes. 47 (2): 35-147.

TURNER, H. (1966): Die globale Hangbestrahlung als Standortsfaktor bei Aufforstungen in der subalpinen Stufe (Stillberg im Dischmatal). Mitt-schweiz.Anst.forstl. Vers'wes. 40 (6): $111-168$.

TURNER, H., ROCHAT, P. \& STREULE, A. (1975): Thermische Charakteristik von Hauptstandortstypen im Bereich der oberen Waldgrenze (Stillberg, Dischmatal bei Davos). Mitt. chweiz.Anst.forstl.Vers'wes. 51 (1): 95-120:

Anschrift des Verfassers:

P. BLASER, Eidg. Anstalt für das forstliche Versuchswesen, 8903 Birmensdorf, $\mathrm{ZH}$, Schweiz. 